\title{
ACTUAR, MAQUILLAR Y ESCRIBIR: TRES PROFESIONES PARA UNA MISMA MUJER, COLETTE
}

\author{
ACTING, MAKING UP AND WRITING: THREE JOBS FOR ONE WOMAN, \\ COLETTE
}

Flavie Fouchard

Universidad de Sevilla

\section{Resumen:}

Sidonie-Gabrielle Colette, también conocida como Colette, fue una reconocida autora francesa. Sin embargo, no solo destacó en la escritura; en este artículo se exploran las disciplinas que abarcó Colette, pasando por el periodismo y la actuación, a partir de documentos tan valiosos como su propia correspondencia, con el fin de redescubrir a esta relevante figura.

Palabras claves:

Colette, escritora, periódico.

\section{Abstract:}

Sidonie-Gabrielle Colette, also known as Colette, was a famous French authoress. However, she did not only stand out with writing; in this article we will explore the different disciplines which Colette took on, such as journalism and performing, from documents as worthy as her own mail, with the aim of rediscover this important figure.

\section{KEY WORD:}

Colette, writer, newspaper. 
"No es verdad

La soledad creador

No está sola

La semilla en la tierra.

Multitudes de gérmenes mantienen

el profundo concierto de las vidas

y el agua es sólo madre transparen te

de un invisible coro sumergido."

Pablo Neruda, "Oda a la soledad", Odas elementales.

Cuando le piden a Colette que hable de poesía en 1938 (conferencia “La poésie que j'aime" en Paysages et Portraits), y como cada vez que le preguntan o piden algo, ella bromea, se divierte tomando el partido opuesto al que se espera de ella. Apreciaremos su sonrisa irónica cuando evoca, regocijándose, a poetas que escribieron prosa y al contrario, a prosistas que escribieron versos. Y más aún cuando reivindica en la misma ocasión, orgullosa, el no haber escrito nunca poesía: ella es prosista de la primera hora y lo será hasta la última. La poesía a finales de siglo XIX es un género muy valorado, quizás el más valorado dentro del ámbito literario: es el género sacrificado por excelencia. El que representa mejor el ideal de creador que se está forjando desde la época romántica y que critica Pablo Neruda en su poema: el creador solitario, alejado del mundo y entregado totalmente a su arte. Añadamos una variable económica: es el género alejado de la lógica mercantil del mundo burgués que lo rodea. En aquel momento los volúmenes de poesía no se vendían, al contrario que la novela que estaba conociendo entonces el crecimiento que sabemos.

Coexistía esta postura vocacional del poeta, que Flaubert encarnó a la perfección y extendió al género novelesco, con la del escritor profesional, mucho menos valorada y representada por otras figuras: la del publicista (el primer marido de Colette fue uno de los más famosos de su época) o la del escritor de novelas por entregas. Pero estos modelos sólo valían para los hombres que se habían apropiado el espacio literario y no estaban dispuestos, en la gran mayoría de los casos, a compartirlo con las mujeres. Las que decidieron escribir tenían que elegir una postura que les permitiera difundir sus escritos y tener audiencia. Varias opciones se les presentaron: calcar su imagen creadora sobre la de los hombres (imitar) o inventarse una, corriendo asimismo el riesgo de no tener audiencia (véase la postura radical de la poeta abiertamente lesbiana Renée Vivien).

El ideal del creador inspirado y solitario no fue nunca la postura adoptada por Colette. Y menos la de la escritora solitaria, sin vida afectiva ni amorosa (Heinich 1996). En ochenta años de vida, focalizando muy a menudo la atención del público, se casó tres veces, tuvo numerosas aventuras con hombres y mujeres, adoptó animales de los más variados (hasta un leopardo), cultivó muchas amistades, mantuvo una correspondencia importantísima. Y también ejerció distintos trabajos a parte de escribir. La cuestión económica parece la de más peso en los discursos de Colette sobre su principal oficio. Prosista, artesana, buena profesional, que participa del circuito económico de publicaciones, en volúmenes, en publicaciones por entregas o en ediciones de lujo ilustradas, en adaptaciones teatrales. Elisabeth Ladenson (2009) insiste en la importancia que tuvo para su carrera el hecho de situarse al margen de modelo masculino (creador inspirado y desinteresado) para poder mantener una postura viable de mujer escritora. Según ella, al no tratar de competir con los autores en su campo, pudo alcanzar la notoriedad. La exageración del aspecto financiero en su discurso sobre la naturaleza de su "necesidad" creadora la puso a salvo y le permitió afianzarse como una de las autoras más relevantes del siglo XX, y eso, en vida. Recordemos que fue la única mujer que mereció el honor de unos funerales nacionales en 1954, después de haber recibido todas las distinciones habidas y por haber de la República.

Sin embargo, la variedad de sus carreras pudo haber sido un gran obstáculo para el reconocimiento de su profesionalidad (sin hablar de su vida sexual, poco convencional, y de sus divorcios). La "pluralidad" de oficios (Heinich 2000): el actuar, a veces semidesnuda para colmo, crear un salón de belleza, escribir en periódicos, participar en proyectos publicitarios, programas radiofónicos etc., podía pasar por diletantismo y no la perjudicó tanto. Frédéric Lefèvre al interrogarla sobre los orígenes de "sus carreras" (Mes apprentissages: 987) en 1937, durante una entrevista radiofónica, nos pone sobre la pista de lo que fascina de Colette: "vous marquez de votre empreinte tout ce que vous touchez. Dans tous les domaines, partout, toujours, vous êtes Colette." (Bonal-RemyBieth 2004: 392). Quizás sea porque Colette la escritora le debió mucho a la actriz, al mimo y a la mujer que decidió poner un negocio de perfumes y productos cosméticos y porque todas tienen en común ciertas características que están relacionadas con la manera que tiene Colette de pensar el oficio de escritora y de organizar su imagen. Una imagen un tanto misteriosa que fascinó a sus contemporáneos gracias a un nombre (firma), un rostro (gracias en gran parte a la fotografía y a su presencia en la prensa), y una voz (con ese acento de Borgoña muy marcado y fácilmente reconocible). Actuar, buscar su inspiración en el "profundo concierto de las vidas" de mujeres y hombres desconocidos, transfigurarse para escribir, "dejarlo" y maquillar a mujeres para retomar el contacto con la vida, independizarse poco a poco sin dejar de ser una misma, reinventándose constantemente, es lo que hizo Colette para encontrar su identidad creadora, y vivir de sus oficios.

\section{Colette escritora profesional}

A lo largo de su carrera en medios públicos, como en su correspondencia, Colette presentó su relación con la escritura como problemática. Repitió que de niña era gran 
lectora pero que nunca sintió la llamada de una vocación y que sin Willy no hubiera escrito ni las Claudine ni sus otras obras, haciendo hincapié en la carga que le suponía el oficio. En agosto de 1933, escribe a Hélène Picard, una de sus amigas íntimas, poeta que vivía en la miseria, a propósito de un libro que tenía que terminar: "C'est dur de ne pas avancer. Peut-être que c'est le commencement de l'impuissance. Avec quelle joie je m'y résignerais, si... j’avais de quoi vivre. Vivre sans écrire, ô merveille ! (Lettres à Hélène Picard: 143). Colette relacionó muy pocas veces escritura y placer. Comentaremos dos. Una vez en La Vagabonde, cuando Renée Néré explica que dejó de escribir para actuar en el music-hall porque la escritura no le daba para vivir y era una actividad de ociosos: “Écrire ! plaisir et souffrance d'oisifs !" (La Vagabonde: 1074). Renée Néré es el único personaje en la obra de Colette que escribe, si no contamos a la narradora Colette y a los escritores reales que describe. En otra ocasión anterior, y esta vez fuera de la ficción, después de haber publicado sus primeros artículos de crítica musical en el periódico La Cocarde en 1895, gracias a Willy y sus consejos, Colette escribe a Charles Maurras, otro escritor francés de la época: "Je danse de joie à la vue de mon nom imprimé." (Brunet-Pichois: 65). Firmaba entonces Colette Gauthiers Villard, el apellido de su padre junto con los de su marido. Para Colette, al contrario que a su madre, el casarse le proporcionó una vía de escape hacia el mundo parisino y la posibilidad de dejar atrás la provincia donde se aburría. Su adaptación en la capital no fue tan difícil como lo dijo años después, construyendo ya el mito de una Colette salvaje, inocente y provinciana, para acentuar el contraste entre ella y Willy, ya marcado por la diferencia de edad. El ámbito literario parisino dejó rápidamente de tener secretos para ella y no dudaba en presentarse como la colaboradora de su esposo (Bonal-Remy-Bieth: 27). Pichois y Brunet sitúan en los años 1900-1903 el momento en que Colette tomó consciencia de su potencial como escritora. La situación de la pareja cambió también por esos años y la necesidad de independizarse de Willy, a nivel afectivo y económico, se hizo sentir. Insisten sobre la herida de Colette como mujer. Esta podría ser una de las razones por las cuales Colette decidió lanzarse al music-hall (foto1), sin dejar de publicar.

Otra razón para empezar una carrera en el music-hall, en el momento de la progresiva separación con Willy, podría hallarse en la relación que mantenía con su madre en aquel momento y que idealizó con el paso de los años. En Sido (1928), Colette comenta la forma que tenía su madre de considerar a sus hijos como genios y de querer encarrilarlos en vías que no necesariamente ambicionaban:

[...] les parents, éblouis les poussent, fût-ce à grands coups de pied dans le derrière, vers des destinées qu ils nomment meilleures. [...] Ma mere qui tenait pour naturel, voir obligatoire, d'enfanter des miracles, professait aussi que "l'on tombe toujours du côté où l'on penche [...] Elle se trompa, nous la trompâmes plus d'une fois. Elle ne se décourageait pas et nous coiffait d'une nouvelle auréole. (Sido: 537)
¿Sólo hablaba aquí Colette de su hermano Léo, soñador empedernido y excelente músico al que su madre ya veía haciendo una carrera brillante que no llevó a cabo, o en estas « destinées qu'ils nomment meilleures » Colette incluye también su propio rechazo hacia el oficio de escritora? Lo cierto es que durante los seis años que duró la carrera de Colette como actriz (1906-1912) Sido no dejó de reprocharle en sus cartas el desperdicio de su talento de escritora en un oficio para el cual no tenía el talento requerido (Bonal, Remy-Bieth 2004: 157) y quizás el arrojarse al teatro no fue sólo una manera de emanciparse de Willy sino también de su madre (veremos más adelante otra razón). No lo sabremos y esto queda como una hipótesis, pero es interesante considerar bajo otra luz la relación real entre madre e hija. Así fue cómo el nombre de Colette se fue haciendo más famoso, debido al teatro en un primer momento, y que a pesar de su creciente reputación como autora, no abandonó rápidamente el apellido de su primer marido, que debía de proporcionarle una notoriedad adicional. Publica en 1904 su primer volumen con el nombre de "Colette Willy" y emplea "Colette (Colette Willy)" hasta 1922. De hecho, Colette reutilizó el nombre de la heroína en el título de su primer gran libro autobiográfico: La Maison de Claudine. En realidad, Colette aprendió mucho de Willy, en cuanto a técnica y comunicación literarias: Willy le enseñó que publicando primero por entregas y más tarde en volumen se gana más dinero, que la publicidad es primordial. El éxito de Claudine se debió en gran parte a la originalidad del tono del texto, pero también a la habilidad de su "autor" para promocionarlo. El nombre de "Claudine", como una marca, designó muchos objetos publicitarios: un tipo de cuello para ropa, un perfume, un sombrero, cigarrillos, un helado en una pastelería de París... Se hicieron adaptaciones teatrales, se editaron postales de Colette, Polaire y Willy. Incluso antes de que la conocieran como actriz y escritora, Colette prestó su rostro y su cuerpo a Claudine.

Ahora y con la distancia que permite examinar la bibliografía completa de Colette, la importancia que tuvo el tema del music-hall en la obra de Colette es evidente, la podemos apreciar en obras como La Vagabonde, L'Envers du Music-hall, Gribiche, Chambre d'hôtel, L'Étoile Vesper, Florie... En 1934, Colette retoca el conjunto que formaban los textos de Les Vrilles de la vigne y coloca el texto « Music-halls » al final, a modo de conclusión. Como lo dicen Brunet et Pichois, el music-hall, el teatro y el mimo inspiraron a Colette y le proporciaonaron materia para su obra, tanto novelesca como periodística. Del mismo modo que le abrieron las puertas del teatro como autora: en 1909 escribió En camarades, obra en dos actos en la que actuó. Más tarde, empezó Chéri como una obra de teatro antes de convertirla en novela y la adaptó con la ayuda de colaboradores, así como La Vagabonde. En ambas obras actuó durante los años veinte, desempeñando el papel de Léa y de Renée Néré 
Insistió mucho sobre su necesidad de ganar dinero. Casarse con Henri de Jouvene no supuso para ella dejar de trabajar; parece que Henry de Jouvenel no tenía mucho más dinero que ella cuando se conocieron y que Colette siguió siendo económicamente independiente. Con su tercer marido, fue aún más difícil porque el negocio en el que trabajaba en los años 30 se vino abajo y parece que después de esto sus obligaciones no estaban tan definidas. Colette tuvo que asumir la carga financiera del matrimonio. Sin embargo, la falta de dinero (y sobre todo el miedo a su falta - muchos biógrafos de Colette ponen de manifiesto que vivía por encima de su nivel de vida) no nos parece que explique por completo la variedad de actividades desarrolladas por Colette durante toda su vida. Hasta sus biógrafos se asombran de ver como a lo largo de los años su actividad fue desbordante e imposible de parar. En la dedicatoria de una foto al final de su vida, Colette escribe: "Le rêve de ma vie ? Et que ferais-je d'un seul rêve ?" Su increíble y siempre renovada curiosidad hacia el mundo, su afán por descubrir, la llevaron por senderos a veces inexplorados o insólitos para una mujer de su generación: como reportera participó en los primeros vuelos de aviones y globos, aprendió a conducir a los 60, se apasionó por el cine desde sus inicios, siendo una de las primeras escritoras en interesarse por este arte y prever la importancia que iba a tener en el siglo. El mundo tuvo mucha más importancia de lo que se dijo en la trayectoria de escritora de Colette. Contrariamente a lo que se piensa sobre ella, se abrió a la modernidad y vivió de pleno la actualidad: viajando mucho, escribiendo sobre la guerra, sobre la moda, las relaciones entre hombres y mujeres a la hora de los cambios sociales provocados por la primera guerra mundial... Ver y describir el mundo desde el punto de vista de lo cotidiano no implicó quedarse al margen de lo que ocurría (aunque su mundo se redujo al final de su vida, especialmente durante la segunda guerra mundial, cuando tenía ya setenta años...). Colette admiró hasta el final estos pequeños acontecimientos con una intensidad que algunos reservaron a las grandes catástrofes del siglo. Nunca dejó de transcribirlos, como impulsada por la fuerza de lo que veía y que nadie parecía contemplar como ella.

\section{2. "Descubrir es uno de mis mayores placeres"}

$\mathrm{Al}$ principio de los años treinta, a pesar del reconocimiento del que goza y en el contexto de la crisis financiera (se vendían menos libros) muchas responsabilidades económicas pesaban sobre Colette. Por otro lado, empieza a sentir cierto hastío hacia la escritura. Maurice Goudeket, su último marido, comentó que después de haber escrito La Fin de Chéri (1926), La Seconde (1929), La Naissance du jour (1928), Sido (1929) y Le Pur et l'Impur (1932), Colette tuvo la sensación de no tener ya nada que decir, de haberse quedado sin palabras y fue entonces cuando decidió, ayudada por el consejo de uno de sus amigos, crear una línea de cosméticos y abrir un salón de belleza para venderlos y maquillar. También parece que este era uno de los sueños de su vida como lo explicó al comentar que siempre había maquillado a sus amigas. Aquí y como siempre, Colette decidió entregarse por completo a su nueva actividad. Diseña ella misma los envoltorios (foto 2), participa en la elaboración de los productos, escoge sus colores corporativos (negro y rojo), diseña el logotipo de la marca: dibuja una caricatura de su rostro con su firma de escritora y sobre todo les pone nombres tan evocadores como los colores que nunca deja de describir en su obra: para barras de labios "cerises volées", "rouge tragique", "pomme d'amour", las cremas: "Je nourris", las sombras de ojos: "Bleu d'orage", "Améthyste"...

Abre una primera tienda en París en 1932 (más tarde pondrá una sucursal en SaintTropez), invitando al todo París para la inauguración. Su invitación reza, provocadora al estilo de Colette: "Êtes-vous pour ou contre le second métier de l'écrivain ?". Colette recibió muchas críticas por este atrevimiento que consistía en rebajar su nombre de escritora utilizándolo para una empresa tan "fútil". La publicidad, muy bien organizada, llevó a mucha gente a la tienda, sobre todo con la curiosidad de acercarse a la escritora. Para promocionar sus productos Colette recorre Francia otra vez, como en el tiempo de sus tournées de los años 1906-12. Durante días se pasa horas maquillando a mujeres, muchas veces anónimas, que acuden a ella. El trabajo es agotador (tiene entonces poco más de sesenta años), pero Colette escribe a Marguerite Moreno desde su gira, el 24 de octubre de 1932: «Ce n'est pas un métier très reposant que je fais. C'est toujours infiniment meilleur que d'être assis devant une feuille de papier, fût-elle bleu turquoise. » (Lettres à Marguerite Moreno: 135). La competencia de las grandes firmas americanas en el sector, en plena expansión en esta época, y sobre todo el hecho de que el negocio se solventaba en gran parte gracias a la figura y la presencia de Colette (las mujeres muy a menudo traían libros para que Colette se los dedicara), la llevaron a abandonar el negocio. Maurice Goudeket no lo recuerda como un fracaso, todo lo contrario, en un momento de sequía y cansancio, le proporcionó a Colette una nueva fuente de inspiración: “L'entreprise avait aidé Colette à vaincre un moment d'aversion pour son métier d'écrivain, tout en la mettant en contact avec un public nombreux, qui lui permettait de renouveler ses thèmes." (Goudeket 1967: 86) Colette tampoco lo consideró como un desperdicio de tiempo ya que en la remodelación de Les Vrilles de la Vigne que mencionamos más arriba, incluye un texto, "Maquillages", que había publicado en una revista en 1933. Éste pinta muy bien lo que le brindó en esos años y el cariño con que lo trata anuncia la comunidad que aspira a formar con las mujeres al final de su vida:

“Je n'ai jamais donné autant d'estime à la femme, autant d'admiration que depuis que je la vois de tout pres, depuis que je tiens, renversé sous le rayon bleu métallique, son visages sans secrets, riche d'expression, varie sous ses rides agiles, $[\ldots] \mathrm{O}$ lutteuses ! [...] L'une s'élance vers mon petit laboratoire comme à une barricade. Elle est mordante, populacière, superbe [...] (Les Vrilles de la vigne: 1006-1007) 
El movimiento del texto reproduce el acercamiento de las generaciones que está realizando Colette en su vejez. Empieza con las recriminaciones de la narradora a su hija sobre su manera de maquillarse. Sigue con un panorama de las formas que tienen de maquillarse las mujeres en función de su edad. Para llegar a la experiencia de Colette en su instituto de belleza, donde pudo comprobar los "milagros" que logra el maquillaje en el estado de ánimo de las mujeres de cincuenta y sesenta años. Colette no es ilusa y señala que este sector mueve tanto dinero como el cine; también sugiere la ambivalencia del maquillaje (motivo sobre el que profundizó en el texto "Bellesde-jours" que sigue a "Maquillages" en la recopilación). Sin embargo le fascina la confianza que le brinda a la mujer y se maravilla de la transfiguración que consiguen los colores: "Nous détenons des armes à enivrer un peintre. » (Les Vrilles de la vigne: 1006). Cuando contempla el rostro de su hija al final del texto, insiste en el poder de los colores y tematiza la descripción con términos de frutas, de flores, borrando el límite entre artificio y naturaleza:

"Pendant que j'écris ma fille est toujours là. Elle a eu un teint de pêche claire, avant de devenir, en dépit de l'hiver, un brugnon très foncé, sous une poudre aussi rousse que le pollen des fleurs du sapin..." (Les Vrilles de la vigne: 1007-8)

La intensidad de la mirada y el afán constante de Colette para captar la realidad y ofrecerla al lector surge, como en "Les Paons" (Prisons et Paradis), donde la descripción se impone a Colette como un reto a su talento, y vemos que los colores llaman de nuevo su atención:

“Je dis «bleu »; mais comment nommer cette couleur qui dépasse le bleu, recule les limites du violet, provoque la pourpre dans un domaine qui est plus mental qu'optique, car si jappelle pourpre une vibration de couleur qui semble franger $\mathrm{ce}$ bleu, je ne le vois pas réellement, je la pressens...O folie de vouloir dépeindre le paon!" (661)

Colette en este texto se interroga ("comment nommer") sobre la manera de poder hacer ver lo que contempla. Cualquier objeto, que podría pasar por insignificante, interesa a Colette. Pero uno de sus temas de predilección es el rostro humano, lugar de tantos dramas y acontecimientos que dejan una huella a la vez efímera y duradera: "Le visage humain fut toujours mon grand paysage" (Trait pour trait). El maquillaje para Colette participó primero en la creación de sus personajes en el music-hall; de él dependía también la calidad del espectáculo (Bella-Vista, "Gribiche": 1171). Para Colette se alzó a otra dimensión (junto con las mismas mujeres) cuando maquilló y se volvió arte gracias a lo que permitía disimular. Un arte del que Colette entendía mucho y valoraba aún más: el maquillarse se transforma en un instrumento de resistencia para la mujer ya que le permite esconder lo que una no quiere enseñar, y le permite guardar su dignidad escondiendo sus penas y sufrimientos. Recordemos que la narradora Colette arremetía contra el « exhibicionismo de la cara » de uno de sus amigos en $L e$ Pur et l'Impur (570). En « Gribiche » (Bella-Vista), un texto de tonalidad autobiográfica, Colette insiste sobre este punto clave para entender su obra: la disimulación del dolor y de las penas y resume lo que le enseñó y permitió actuar: “Des mois passèrent, et des années, pendant lesquels, me donnant ça et là en spectacle, j'usais du droit de me taire sur moi-même." (Bella-Vista, "Gribiche": 1353). Callar lo que más le atañe, esconderse detrás de representaciones o de sus personajes (Claudine, Léa, numerosas Colette narradoras y personajes...), este es uno de los resortes de la fascinación que ejerció Colette.

\section{Mostrar y esconderse o el arte de fascinar}

No es casualidad, Colette eligió publicar bajo el nombre de La Femme cachée (1924) una de sus recopilaciones de relatos cortos y cuentos. La dialéctica entre el ponerse en escena y esconder lo más hondo de ella parece regir toda la obra de Colette y su relación con el público. Pronto empezó poniendo de manifiesto su voluntad de ser dueña de lo que circulaba sobre ella. Desde muy temprano Colette utilizó la prensa para luchar contra la hipocresía de la burguesía en cuanto a la doble moral relacionada con el divorcio y el matrimonio. Lo hizo escribiendo artículos donde recreó diálogos ficticios con su "amiga Valentine" arquetipo de la burguesa (Paysages et Portraits, Contes des mille et un matins, Les Vrilles de la vigne) así como cartas a los periódicos para intentar controlar la imagen que se da de ella. "De quoi est-ce qu'on a l'air ?" (publicado en el periódico La Vie Parisienne el 25 de enero de 1908 y recopilado en Les Vrilles de la vigne) es uno de ellos. Trató con mucha ironía el poder de la mirada y el control ejercido por los otros en una clase social. A partir de un ejemplo muy concreto el poder levantarse a la hora que le apetece mientras que a su amiga Valentine le es imposible por miedo al "qué dirán". Colette denunciaba el condicionamiento de la opinión de los miembros de su clase social. En aquel momento de su vida Colette se negó a ceder ante la presión del ideal de la mujer burguesa, pero no renunció sin embargo a tener una imagen pública. De hecho, su imagen era bastante mala en ésa época y le costó mucho atenuar la reputación que tenía. Lo hizo gracias a la fotografía: Colette es la escritora francesa (escritores incluidos) más fotografiada, y de lejos (Ducrey 2004). Los retratos la representan a menudo a su mesa de trabajo, posando como escritora y casi todos son poses y no instantáneas. Y gracias a su presencia en los periódicos, como periodista, y reivindicando su libertad y su estatuto de creadora. Tomaremos tres ejemplos que muestran perfectamente que Colette, si escribió a partir de sus vivencias, no consideraba su oficio como mero registro de ellas y que aspiraba a ser leída en función de criterios estéticos. Veamos primero su voluntad de controlar lo que se proyecta de su vida privada en el momento de su primera separación, de la 
que se hablaba en todos los periódicos de la época. En numerosos artículos se burlaban de su nueva pareja oficial, la Marquesa de Belbeuf (Missy). En esta ocasión, en 1906, no dudó en escribir una carta a uno de estos periódicos, para quejarse de una crónica sobre las parejas que formaban Willy con otra mujer y ella con Missy - parece que el periódico los consideraba sospechosos de mantener relaciones mal delimitadas: “Ne réunissez pas si... intimement dans l'esprit de vos nombreux lecteurs [ironie !], deux couples qui ont arrangé leur vie de la façon la plus normale que je sache, qui est celle de leur bon plaisir." (Brunet, Pichois 1999: 133). En 1909, cultiva su imagen de mujer a quien no le gusta opinar sobre temas generales: “Je n'aime pas beaucoup les idées générales" en una carta a la revista Comoedia, publicada en el número 4 del 15 de febrero de 1909, para contestar a un artículo del crítico Gaston de Pawlowski sobre la representación de su comedia En Camarades. El periódico la titula así: “Une lettre de Colette Willy. À propos de ses débuts comme auteur dramatique, actrice et danseuse." En el negarse a responder a los críticos que le preguntaban por su opinión acerca del teatro, de la literatura en general, podemos reconocer esta voluntad de construir su imagen al margen del mundo artístico que señaló Elisabeth Ladenson. Brunet y Pichois (1999: 216-217) señalan otra carta de Colette a un periódico para ir en contra de la presentación que la revista Femina hizo de su artículo "Maternité" (Paysages et Portraits) en el número del 15 de enero de 1914. Los autores de la revista, en la presentación del artículo y para anunciar el inicio de la colaboración de Colette, añadieron este título: "Impressions d'une maman. Les premières heures" informando al lector de que Colette acababa de dar a luz a su hija. El director publicaría la carta de Colette el 1 de febrero. El tono es tajante, y muy parecido al de las que leímos anteriormente:

\section{Que je sois mère, cela ne regarde pas le lecteur. Je lui donne une œuvre que je souhaite littéraire, c'est l'auteur qui paraît devant lui, ce n'est pas la femme, et s'il a le droit de me juger comme écrivain, son droit s'arrête là. [...] Il y a là une nuance, et un peu plus qu'une nuance. Vous êtes trop fin, cher ami, pour ne pas l'avoir} aperçue.

Colette aparece firme, autoritaria y deseosa de dejar las cosas claras, imagen que no corresponde a la timidez que se le atribuye en sus primeros años pasados en París. Si estas cartas nos desvelan una Colette paradójicamente preocupada por mantener su vida privada a distancia, también nos revelan que atribuía a sus artículos el mismo valor que a su obra literaria, aunque eran trabajos alimenticios. Los escribía la misma autora, Colette. Demuestran que tenía en alta consideración todos sus trabajos de escritura, aunque se negara a opinar sobre la creación en general. Del mismo modo, afirmó muchas veces que no le gustaban las ideas generales pero no dejó de dar su opinión sobre los temas más variados en la prensa. Sus crónicas, escritas bajo los títulos
"Mon journal”, “Le journal de Colette”, “La chronique de Colette”, si se inspiraban de sus experiencias, no eran diarios donde contara su vida. Brunet y Pichois concluyen sobre su actividad periodística:

Ainsi, pendant plus de trente ans, Colette, égocentrique mais pourtant peu "diariste », aura tenu un journal dans la presse, dans lequel elle aura exprimé son opinion sur de nombreux sujets ( « L'Opinion d'une femme » fut le titre d'une de ses chroniques), mais elle n'aura jamais tenu un journal personnel, malgré les titres qu'elle a utilisés : Journal intermittent, Journal à rebours. (1999: 214)

La postura de Colette fue mucho más reflexiva y ambigua de lo que ella afirmara Incluso podríamos decir que entraron en su construcción elementos que pertenecían a la figura idealizada del creador romántico: marginal y doliente. En otra carta a Marguerite Moreno, Colette se queja de nuevo del proceso de escritura: “C'est une lutte si sombre, celle qui use une griffe sur un papier. Et sans témoins, et sans soigneurs. Et sans passion. Et il y a la source bleue à côté, l'appel des paons, l'odeur de l'air. C'est très triste." (Lettres à Marguerite Moreno, 14 de junio de 1926, 118). Ahora bien, para Colette, un placer tampoco llega siempre puro de dolor. En La Vagabonde, texto en el cual la narradora menciona la "volupté d'écrire" (1084), se queda ella pensando en otro momento, en su próximo "naufragio", en su entrega al placer. En la ensoñación de la narradora, la tristeza aparece primero como consecuencia del placer: "je regarde avec terreur s'approcher la tristesse aux douces mains puissantes, guide et compagne de toutes les voluptés..." (La Vagabonde: 1110). La tristeza es la tonalidad que le da a su texto-meditación sobre los sentidos y el placer sexual: Le Pur et l'Impur (566). Nathalie Heinich explica que "la reconnaissance de la douleur est ce qui autorise l'écart de grandeur " (Heinich 2005: 43), y Colette escenificó este dolor al presentar siempre el escribir como una tarea impuesta, una costumbre dolorosa. También asumió una postura de distancia con respecto a otros creadores, una postura marginal: en la ya mencionada conferencia sobre la poesía se señaló como una excepción: “vous avez devant vous un individu d'une espèce extrêmement rare, une sorte de monstruosité, un prosateur qui n'a jamais écrit de vers." (Paysages et Portraits: 202). Y sobre todo se diferencia de su propio sexo, apropiándose de la figura andrógina que utilizaron numerosos autores de su época, incluido Flaubert (Fabre 2000). Lo hace en Le Pur et l'Impur al reflexionar sobre su identidad que califica de "véridique hermaphrodisme mental" (586). En este mismo texto, a parte de su constitución particular, excepcional, sobre todo en una mujer, se identifica con los "monstruos" de los espacios que describe, en particular con los hombres homosexuales, de quienes admiró... el "arte de fingir" (Le Pur et l'Impur: 642). En este texto de Colette, que no se valora tanto para examinar su postura creadora como las obras relacionadas con su infancia y con su madre, la tensión entre el arte de representar y de esconder encuentra su expresión máxima: la 
figura del velo es omnipresente, el tono ironico abunda, nunca se está muy seguro de lo que opina la narradora y la autora se niega a definir lo que entiende por "puro" en la conclusión, cuando su título la instaba a hacerlo. Este arte se encuentra relacionado con el don de la autora para encontrar manantiales ocultos (Le Pur et l'Impur: 643). Es decir, para llegar al "invisible coro sumergido" del que nos habla Neruda a partir de la realidad, impura por naturaleza, pero que no dejar de entrañar un misterio digno de interés.

La "vocation" (La Naissance du jour: 304) de Colette, la "capteuse de sources" (Le Pur et l'Impur: 643) se atisba ahora: un sentido muy fuerte de la profesionalidad, un deber de describir de la mejor manera posible el asombro cotidiano frente al mundo y la magia que se desprende de la realidad. La propia identidad de la autora está en juego en este trabajo que implica transfigurar lo cotidiano gracias a una mirada penetrante y curiosa de todo lo que puede pasar por más anodino. La verdad no se encuentra siempre en lo evidente para Colette: “Ce n'est pas dans la zone illuminée que se trame le pire..." (La Naissance du jour: 316) Como tampoco los escritos de una mujer se pueden reducir a obras "fatalement autobiographiques" (La Naissance du jour: 316). Sin embargo nosotros, lectores ávidos, la seguimos buscando entre sus fotografías, sus novelas, sus relatos cortos, su crítica teatral, sus entrevistas con periodistas, sus cartas, siempre convencidos de que no se desvela del todo, de que queda otra cosa por descubrir que no enseñó, y fascinados por esta manera de crear y reinventar su propio personaje a lo largo de toda una vida. Escuchemos a Colette, personaje de $L a$ Naissance du jour, en una conversación con Anna de Noailles, la poetisa contemporánea de Colette más conocida en su época, totalmente solidaria de la postura romántica e inspirada:

"Vous n'aimez donc pas la gloire ? " me demandait Mme de Noailles. Mais si. Je voudrais laisser un grand renom parmi les êtres qui, ayant gardé sur leur pelage, dans leur âme, la trace de mon passage, ont pu follemer seul moment, que je leur appartenais. (La Naissance du Jour: 304 )

Siempre tan perspicaz y consciente de los mecanismos que unen al público y al creador, Colette nos revela aquí una de las razones de su éxito y de la fascinación que sigue ejerciendo: la ilusión de poseerla, subrayando la importancia del "deseo del autor" en su lector, y revelando que tenía una conciencia aguda de lo que se ponía en
Brunet, A. y Pichois, C., Colette, Paris, Éditions de Fallois, 1999, 602 p.

Colette, Lettres de la Vagabonde, texte annoté et établi par Claude Pichois et Roberte Forbin Flammarion, Paris, 1961, 293 p.

----., Lettres à Hélène Picard (LHP), Lettres au Petit Corsaire, Euvre complète, texte établi et annoté par Claude Pichois, Genève, Édition Crémilles, 1972, 319 p.

----, Lettres à Marguerite Moreno, texte établi et annoté par Claude Pichois, Édition Crémilles, Genève, 1972, $325 \mathrm{p}$.

----, La Vagabonde, Euvres, tome I, Gallimard, « Bibliothèque de la Pléiade », 1984.

----, Les Vrilles de la vigne, Euvres, tome I, Gallimard, « Bibliothèque de la Pléiade », 1984.

----, La Naissance du jour, CEuvres, tome III, Gallimard, « Bibliothèque de la Pléiade », 1991.

----, Le Pur et l'Impur, Gallimard, « Bibliothèque de la Pléiade », tome III, 1991.

----, Mes apprentissages, Euvres, tome III, Gallimard, «Bibliothèque de la Pléiade », 1991.

----, Paysages et Portraits, textes établis, présentés et annotés par Marie-Françoise BerthuCourtivron, Flammarion, 2002, 370 p.

Ducrey, G., "Colette et la photographie" dans Notre Colette, sous la direction de Kristeva, J., P.U.R., Rennes,2004, pp. 92-105

Fabre, D., L'androgyne fécond ou les quatre conversions de l'écrivain, CLIO. Histoire, femmes et sociétés, en línea, 11, 2000, mis en ligne le 24 mai 2006. URL: http://clio.revues.org/index214. html. Consultado el 20/09/10.

Goudeket, M., Près de Colette (1956), Le cercle du bibliophile, 1967, 251 p.

Heinich, N., États de femme, l'identité féminine dans la fiction occidentale, Paris, 1996, Gallimard, NRF essais, $397 \mathrm{p}$.

----, Être écrivain Création et identité, Paris, La Découverte, 2000, 368 p.

----,L'Éliteartistique Excellenceetsingularitéen régimedémocratique,Paris, NRFGallimard,2005,370p. Izquierdo, P., Devenir poétesse à la Belle Époque 1900-1914, Étude littéraire, historique et sociologique, Paris, L'Harmattan, 2009, 396 p.

Ladenson, E., «Colette et le XIXe siècle », Colette : complexités et modernités, Cahiers Colette no31, 2009 pp. 23-33.

Marks, E. y Strambolian, G., Homosexualities and French Literature Cultural Contexts, Critical Texts, Ithaca and London, Cornell University Press, 1979, 387 p.

Virmaux, A. y Brunet, A., Colette et le cinéma, France, Librairie Arthème Fayard, 2004. juego durante la lectura.

\section{REFERENCIAS BIBLIOGRÁFICAS}

Bonal, G. y Remy-Bieth, M., Colette intime, Paris, Phébus, 2004, 446 p 